\title{
EUS-guided radiofrequency ablation of small pancreatic adenocarcinoma: a new therapeutic option for patients unfit for surgery
}

\author{
Dario Ligresti, MD, Michele Amata, MD, Luca Barresi, MD, Mario Traina, MD, Ilaria Tarantino, MD
}

Radiofrequency ablation (RFA) is a well-established therapeutic option for ablation of dysplastic and neoplastic tissue by the use of local thermal coagulative necrosis. The percutaneous approach has been shown to be effective and safe, but it is unfeasible in cases of lesions with interposition of organs and/or vessels. For pancreatic lesions, surgery-assisted RFA has been described as an invasive treatment for locally advanced pancreatic adenocarcinoma, with a substantial risk of thermal injuries to major vessels or adjacent structures. EUS-guided RFA is a minimally invasive approach that offers high-quality real-time imaging guidance during selective ablation of pancreatic lesions. ${ }^{1-4}$ It has been shown to be feasible and safe in patients with unresectable pancreatic cancer., ${ }^{5,6}$ In this video (Video 1, available online at www.VideoGIE.org), we present a case of EUS-guided RFA of a resectable small pancreatic adenocarcinoma in a patient unfit for surgery.

An 83-year-old patient with an incidental CT finding of a 15-mm solid pancreatic lesion (Fig. 1) underwent contrastenhanced (CE) EUS, which revealed a 15-mm hypoechoic and hypoenhancing lesion of the neck of the pancreas, with irregular borders (Fig. 2A and B). EUS fine-needle biopsy was performed with a 20-gauge fenestrated needle, and the lesion was diagnosed as an adenocarcinoma. Although there was no vascular invasion or distant metastases, the patient was judged unfit for surgery because of multiple severe cardiovascular comorbidities. The patient was then referred for EUS-guided RFA of the lesion.

We used a novel EUS-RFA system (STARmed, Seoul, Korea) consisting of a 19-gauge needle electrode (EUSRA, EUS-guided RFA electrode) covered with a sheath, except for the terminal $1 \mathrm{~cm}$ with a sharp conical tip for energy delivery, and an RF generator. Furthermore, this system features an internal cooling system that circulates chilled saline solution through the needle electrode during the RFA procedure to prevent charring of the surface of the electrode and to improve the accuracy of the ablation. The lesion was punctured at 2 different points, and $20 \mathrm{~W}$ ablation power was delivered at both sites under direct EUS control (Fig. 2C). Ablation time was related to tissue impedance measured in real time by the RF generator. Bioimpedance reflects the property of a tissue to conduct electricity. An impedance rise is an indirect sign of cellular disruption, tissue charring, and hampering of electrical conduction. ${ }^{5}$ When a sudden rise in impedance was registered, power delivery was then stopped.

After RFA, control CE-EUS in the same session showed no captation of the ablated area (Fig. 2D). The patient's postprocedural course was uneventful, and no early or delayed adverse events occurred. Magnetic resonance imaging follow-up at 3 months revealed a $2.4-\mathrm{cm}$ avascular area and RFA-related necrosis (Fig. 3). The patient was still alive 12 months after discharge and in good overall health.

RFA is an effective technique for neoplastic tissue ablation. In GI endoscopy, its use was initially studied for the treatment of dysplastic Barrett's esophagus, which remains one of its main applications. ${ }^{4}$ Beyond Barrett's esophagus, endoscopic indications for RFA are rapidly evolving, with encouraging results. ${ }^{7-9}$ EUS-guided RFA offers, with minimal invasiveness, the best real-time visualization of pancreatic tissue, also offering the possibility to accurately study relations to adjacent vessels by Doppler scanning. Some animal studies assessing feasibility and safety of EUSguided RFA of pancreatic tissue have been published. ${ }^{10-13}$ Human studies on EUS-guided RFA of the pancreas are

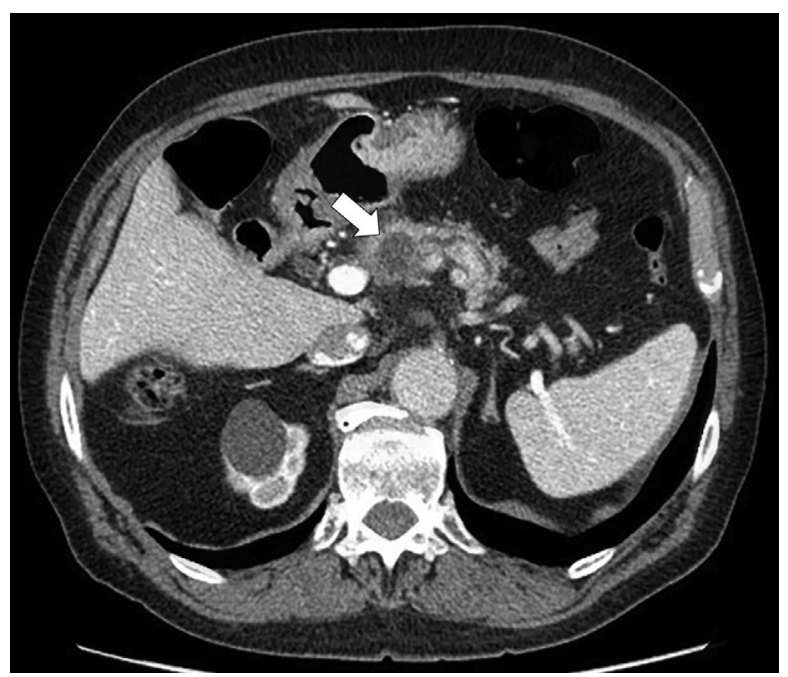

Figure 1. CT scan showing a low-density mass at the neck of the pancreas (arrow) 

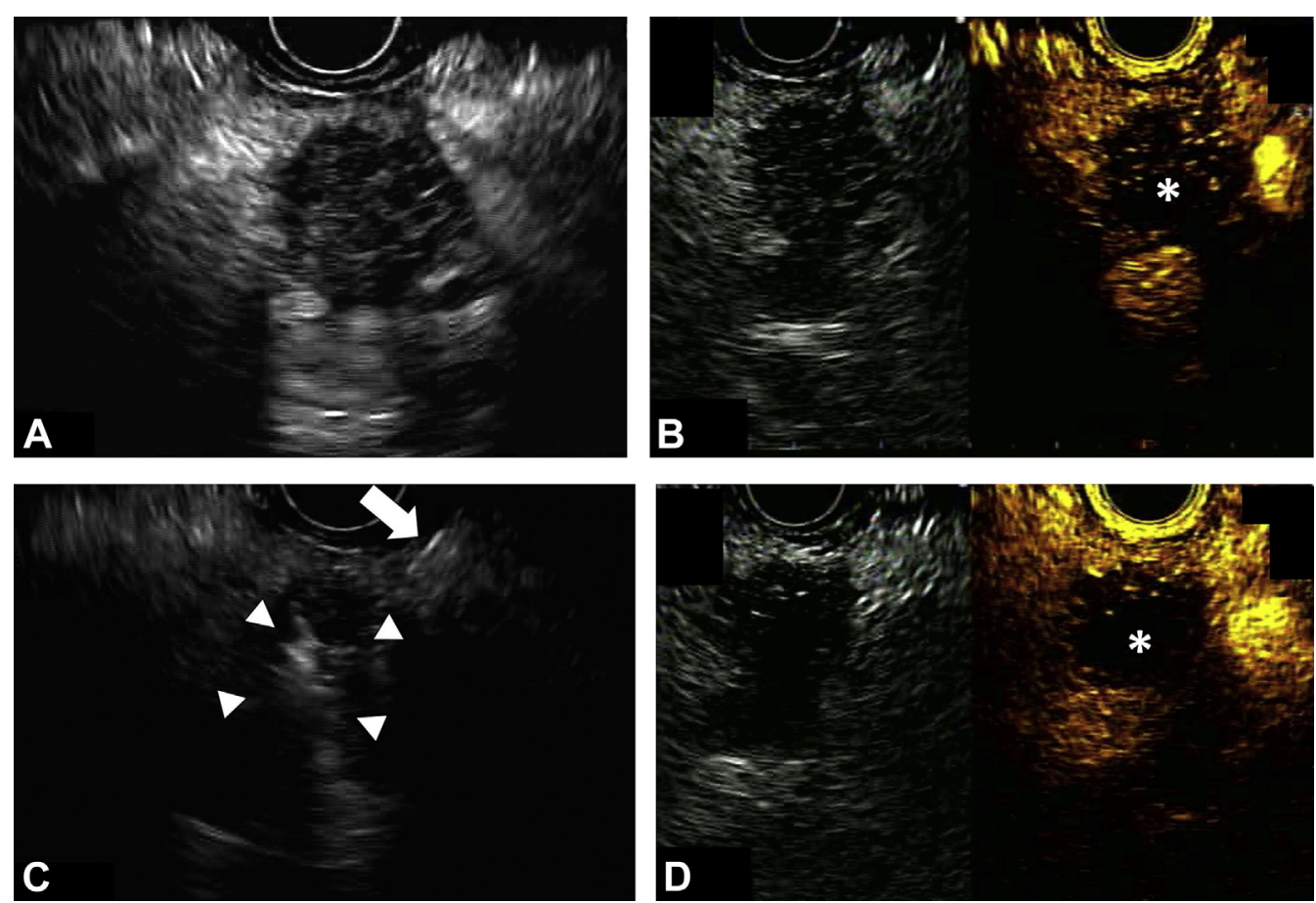

Figure 2. A, EUS view of a 15-mm hypoechoic lesion with irregular border of the neck of the pancreas. B, Contrast-enhanced EUS (CE-EUS) showing hypoenhancement of the lesion $(*)$. C, Delivery of $20 \mathrm{~W}$ ablation power with the EUS-guided radiofrequency ablation (RFA) 19-gauge needle electrode (arrow). Echogenic bubbles start appearing around the needle tip, indicating completeness of RFA at the site (arrowheads). D, Immediately after RFA, CE-EUS view showing no captation of the ablation area $(*)$.

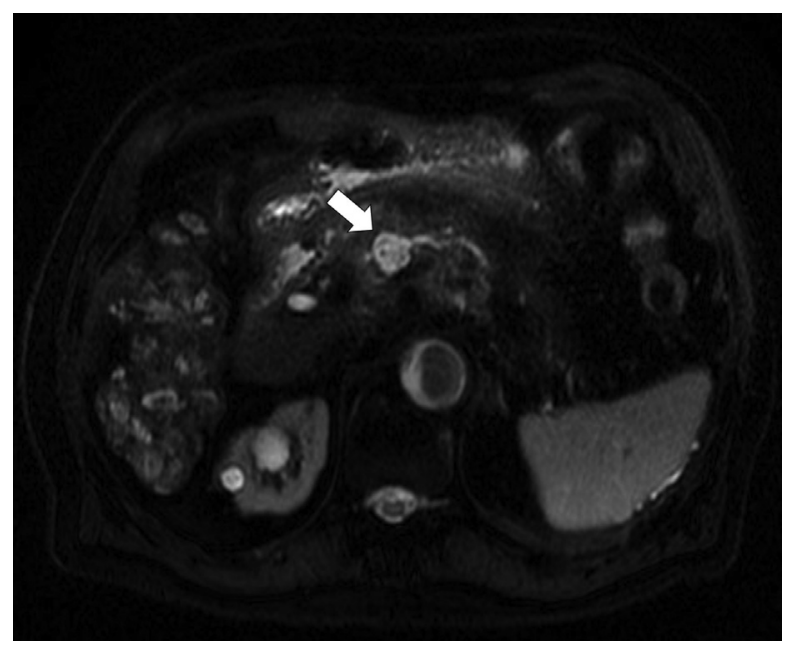

Figure 3. Three months after radiofrequency ablation (RFA), magnetic resonance image showing low-density RFA-related necrotic area (arrow).

limited and refer to a heterogeneous group of lesions (neuroendocrine tumors and pancreatic cystic neoplasms) in patients unfit for surgery or who refuse it. ${ }^{1,2}$ Two other articles on unresectable pancreatic cancer in humans have been published, demonstrating the feasibility and safety of the EUS-guided technique. ${ }^{4,5}$

Surgery remains the treatment of choice for resectable pancreatic cancer. However, we found that EUS-RFA of a resectable pancreatic adenocarcinoma in a patient unfit for surgery was a feasible procedure and that it could perhaps become an emerging field in such patients. Further studies will be needed to define the long-term outcomes.

\section{DISCLOSURE}

All authors disclosed no financial relationships relevant to this publication.

Abbreviations: CE-EUS, contrast-enhanced EUS; RFA, radiofrequency ablation.

\section{REFERENCES}

1. Lakhtakia S, Ramchandani M, Galasso D, et al. EUS-guided radiofrequency ablation for management of pancreatic insulinoma by using a novel needle electrode (with videos). Gastrointest Endosc 2015;83:234-9.

2. Pai M, Habib N, Senturk H, et al. Endoscopic ultrasound guided radiofrequency ablation, for pancreatic cystic neoplasms and neuroendocrine tumors. World J Gastrointest Surg 2015;7:52-9.

3. Armellini E, Crinò SF, Ballarè M, et al. Endoscopic ultrasound-guided radiofrequency ablation of a pancreatic neuroendocrine tumor. Endoscopy 2015;47:E600-1.

4. ASGE Technology Committee; Navaneethan U, Thosani N, Goodman A, et al. VideoGIE 2017;2:252-9.

5. Song TJ, Seo DW, Lakhtakia S, et al. Initial experience of EUS-guided radiofrequency ablation of unresectable pancreatic cancer. Gastrointest Endosc 2016;83:440-3.

6. Scopelliti F, Pea A, Conigliaro R, et al. Technique, safety, and feasibility of EUS-guided radiofrequency ablation in unresectable pancreatic cancer. Surg Endosc. Epub 2018 May 15. 
7. Mok SRS, Khara HS, Johal AS. Cholangioscopic appearance after radiofrequency ablation of cholangiocarcinoma. VideoGIE 2017;2:279-83.

8. Inderson A, Slingerland M, Farina Sarasqueta A, et al. EUS-guided radiofrequency ablation for a left adrenal oligometastasis of an esophageal adenocarcinoma. VideoGIE 2018;3:159-61.

9. Attili F, Boškoski I, Bove V, et al. EUS-guided radiofrequency ablation of a hepatocellular carcinoma of the liver. VideoGIE 2018;3:149-50.

10. Goldberg SN, Mallery S, Gazelle GS, et al. EUS-guided radiofrequency ablation in the pancreas: results in a porcine model. Gastrointest Endosc 1999;50:392-401.

11. Kim HJ, Seo DW, Hassanuddin A, et al. EUS-guided radiofrequency ablation of the porcine pancreas. Gastrointest Endosc 2012;76:1039-43.

12. Silviu UB, Daniel P, Claudiu M, et al. Endoscopic ultrasound guided radiofrequency ablation of the pancreas: an experimental study with pathological correlation. Endosc Ultrasound 2015;4:330-5.
13. Gaidhane M, Smith I, Ellen K, et al. Endoscopic ultrasound guided radiofrequency ablation (EUS-RFA) of the pancreas in a porcine model. Gastroenterol Res Pract 2012;2012:431451.

Endoscopy Service, Department of Diagnostic and Therapeutic Services, Istituto Mediterraneo per i Trapianti e Terapie ad alta specializzazione, Palermo, Italy.

Copyright (c) 2018 American Society for Gastrointestinal Endoscopy. Published by Elsevier Inc. This is an open access article under the CC BYNC-ND license (http://creativecommons.org/licenses/by-nc-nd/4.0/).

https://doi.org/10.1016/j.vgie.2018.09.008

\section{Submit to VideoGIE}

VideoGIE is now indexed in PubMed Central.

VideoGIE is an Open Access, online-only journal indexed in PubMed Central. Submit video cases of endoscopic procedures used in the study, diagnosis, and treatment of digestive diseases.

VideoGIE publishes the following article types:

- Case Reports: Reports of the diagnosis and management of digestive diseases using a single case.

- Case Series: Reports of the diagnosis and management of digestive diseases using 3 or more cases.

- Tools and Techniques: Educational videos demonstrating the use of a particular endoscopic tool or technique. The goal of this section is to help trainees, endoscopy nurses, and technicians learn how best to use the tools of endoscopy for high-quality care.

All manuscripts must be submitted online at http://www.editorialmanager.com/vgie 